\title{
Retraction Note to: Lactoferrin promote primary rat osteoblast proliferation and differentiation via up-regulation of insulin-like growth factor-1 expression
}

\author{
Jian-ming Hou ${ }^{1,2} \cdot$ Man $\mathrm{Wu}^{2} \cdot$ Qing-ming $\operatorname{Lin}^{2} \cdot$ Fan $\mathrm{Lin}^{3} \cdot \mathrm{Ying} \mathrm{Xue}^{3}$. \\ Xu-hua Lan ${ }^{1} \cdot$ En-yu Chen ${ }^{2}$ Mei-li Wang ${ }^{2}$ Hai-yan Yang ${ }^{2} \cdot$ Feng-xiong Wang ${ }^{2}$
}

Published online: 18 August 2015

(C) Springer Science+Business Media Dordrecht 2015

Retraction Note to: Mol Biol Rep (2014) 41:5019-5030

DOI 10.1007/s11033-014-3368-2

The Publisher and Editor retract this article in accordance with the recommendations of the Committee on Publica tion Ethics (COPE). After a thorough investigation we have strong reason to believe that the peer review process was compromised.

The online version of the original article can be found under doi:10.1007/s11033-014-3368-2.

Jian-ming Hou hjm996@126.com

1 Endocrinology Department, Fujian Provincial Hospital, No. 134 Dong Jie Road, Fuzhou 350001, China

2 Provincial Clinical Medical College of Fujian Medical University, Fuzhou 350004, China

3 Cadre Clinic 1 Department, Fujian Provincial Hospital, Fuzhou 350001, China 